\title{
Reforma y gobernanza en salud: el atractivo del gerenciamiento en la acción pública
}

\section{Governance and Health: The rise of the managerialism in public sector reform*}

\author{
Jean L. Denis ${ }^{1}$, Lise Lamothe ${ }^{1}$, Ann Langley² y Stéphane Guérard ${ }^{3}$
}

1 Départemente d'administration de la Santé, Université de Montréal, Canadá. jeanlouis.denis@umontreal.ca, lise.lamothe@umontreal.ca

2 Département de Management, École des Hautes Études Commerciales, Montréal. Canadá. ann.langley-laporte@umontreal.ca

3 Institute of Organization and Administrative Science, University of Zurich. Suiza. stephane.guerard@iou.uzh.ch

Recibido 6 Febrero 2010/Enviado para Modificación 10 Febrero 2010/Aceptado 24 Febrero 2010

\section{RESUMEN}

El artículo propone un examen de diferentes proyectos de reforma de los sistemas de salud de Canadá y de algunas provincias canadienses. Indica ciertas tendencias en la renovación de la gobernanza. El análisis se apoya sobre la hipótesis de que una reforma es per se un ejercicio de renovación de la concepción y de las prácticas de gobernanza. Al buscar renovar la gobernanza, los reformadores esperan encontrar instrumentos suficientes y eficaces para alcanzar los objetivos anunciados en sus reformas. El artículo muestra que la concepción y las modalidades operativas de la gobernanza se transforma a través del tiempo y que ellas muestran ciertas tensiones inherentes al proceso de transformación y legitimación del sistema público de salud. La primera parte discute las relaciones entre reforma y cambio. La segunda describe la noción de gobernanza implícita en el análisis. La tercera parte traza la evolución de la concepción de gobernanza, mediante el análisis de contenido de diferentes informes para propuestas de reformas Se analizan las ideologías y los principios organizadores del núcleo de los proyectos reformistas, para dar cuenta de las nuevas tendencia en materia de gobernanza reveladas por las reformas. Se identifican cinco ideologías: la ideología democrática, la ideología de la "salud poblacional", la ideología privatizadora o de negocios, la ideología gerencial y la ideología de la equidad y del humanismo. Ello permite una discusión sobre el alcance de la perspectiva gerencial de la gobernanza que parece dominar los actuales proyectos de reforma.

Palabras Clave: Gobernanza, gobierno, reforma, sistemas de salud (fuente: DeCS, BIREME).

*El presente texto fue tomado y traducido de la obra original con el permiso de Les Presses de l'Université Laval: Denis JL, Lamothe L, Langley, A, Guérard S. Réforme et Gouverne en Santé. L'attrait pour une managérialisation de l'action publique. In : Laborier P, Noreau G, Rioux M, Richard G (Dir). Les Réformes en Santé et en Justice. Le Droit et la Gouvernance.Presses de l'Université de Laval, 2005.

Traducción: Carmen Rico de Sotelo. Doctora en Ciencias de la Información, Profesora titular de la Universidad de Quebec en Montreal. Enero 2010 


\section{ABSTRACT}

The article examines various healthcare systems reform projects in Canada and some Canadian provinces and reveals some tendencies in governance renewal. The analisis is based on the hypothesis that reform is an exercise aiming at the renewal of governance conception and practices. In renewing governance, reform leaders hope to use adequate and effective levers to attain announced reform objectives. The article shows that the conceptions and operational modalities of governance have changed over time and that they reveal tensions inherent to the transformation and legitimation process of public healthcare systems. The first section discusses the relationships between reform and change. The second section defines the conception of gouvernance used for the analisis. Based on a content analisis of the various reform reports, the third section reveals the evolution of the conception of governance in healthcare systems in Canada. In order to expose the new tendencies, ideologies and operational principles at the heart of the reform projects are analysed. Five ideologies are identified: the democratic ideology, the "population health" ideology, the business ideology, the managerial ideology and the ideology of equity and humanism. This leads to a discussion on the dominant influence of the managerial ideology in the current reform projects.

Key Words: Governance, reform, healthcare systems (source: MeSH, NLM).

\section{RESUME}

L'article propose un examen de différents projets de réforme des systèmes de santé au Canada et dans certaines provinces canadiennes. II révèle certaines tendances dans le renouvellement de la gouvernance. L'analyse s'appuie sur l'hypothèse qu'une réforme est en soit un exercice de renouvellement de la conception et des pratiques de gouvernance. En cherchant à renouveler la gouvernance, les réformateurs espèrent se donner des leviers suffisants et efficaces pour atteindre les objectifs annoncés des réformes. L'article montre que les conceptions et les modalités opératoires de la gouvernance se sont transformées à travers le temps et qu'elles révèlent certaines tensions inhérentes au processus de transformation et de légitimation des systèmes publics de santé. Une première section discute des rapports entre réforme et changement. Une deuxième section décrit la conception de la gouvernance à la base de l'analyse. La troisième section retrace au moyen d'une analyse du contenu de différents rapports proposant des réformes, l'évolution de la conception de la gouvernance. Pour rendre compte des nouvelles tendances en matière de gouvernance révélées par les réformes, les idéologies et les principes organisateurs qui sont au cœur des projets réformistes sont analysés. Cinq idéologies peuvent être dégagées: l'idéologie démocratique, l'idéologie "santé des populations ", l'idéologie affairiste, l'idéologie managériale et l'idéologie de l'équité et de l'humanisme. Ceci permet une discussion sur la portée d'une approche dite managériale de la gouvernance qui semble dominer les projets de réforme actuels.

"Otra opinión que circula, y que es aún más preocupante, es que nuestro sistema de salud está dirigido por una suerte de "piloto automático" que debe continuar su curso en forma inmutable. A mi entender, esta opinión menosprecia el ingenio y el espíritu de innovación que ha caracterizado desde hace mucho tiempo la manera de obrar canadiense. Es un punto de vista erróneo y desprovisto de fundamento. Los gobiernos pueden elegir opciones acertadas en las áreas en 
donde deben invertir y sobre los instrumentos a priorizar; pueden modificar las orientaciones actuales en materia de gastos. Mejores prácticas de gestión, instituciones más flexibles y cooperativas, y un acento más marcado en la prevención pueden permitir economizar importantes sumas de dinero..."(1). "Creemos que la gestión es una función esencial que ha estado demasiado tiempo desvalorizada en el funcionamiento de los servicios sociales y de salud. Es necesario inyectar fuertes dosis de gerenciamiento en el sistema. Ninguna organización puede sobrevivir y desarrollarse sin jefes competentes, motivados por el logro de resultados y que dispongan de márgenes de acción suficientes"(2).

$\mathrm{E}$ stas dos citas extraídas de informes de comisiones $(1,2)$ sobre el futuro de los sistemas de salud demuestran de forma ejemplar hasta qué punto la cuestión de la gobernanza está en el núcleo de las reformas. La primera cita pertenece a la carta introductoria al informe de la Comisión sobre el futuro del sistema de salud en Canadá (1) y subraya, al menos desde el punto de vista de sus autores, que una condición fundamental para la viabilidad de los sistemas públicos de salud de los Estados modernos es su capacidad para introducir cambios apropiados, deliberados, y en el momento oportuno, sin desestabilizar a ultranza esos sistemas. La segunda cita extraída del Informe de la Comisión de estudio sobre la salud y los servicios sociales de Québec (2) da cuenta de la voluntad de tomar las riendas de los problemas del sistema de salud y de apelar a una gestión más vigorosa para resolver sus disfuncionamientos. En los dos casos, la reforma es, a la par, un ejercicio de concepción y de aplicación de soluciones para incrementar el buen funcionamiento del sistema de salud, y en definitiva, de garantizar su legitimidad. La reforma apela simultáneamente a una voluntad de actuar en un espacio estructurado por valores que se suponen consensuados y a una búsqueda de soluciones racionales a problemas prácticos. La gobernanza consiste en un conjunto de ideas, de normas, de recursos y de procesos que son movilizados por los reformadores para inducir en forma deliberada cambios en el sistema de salud.

El examen de diferentes proyectos de reforma de los sistemas de salud en Canadá y en algunas provincias canadienses revela ciertas tendencias en la renovación de la gobernanza. De hecho, planteamos la hipótesis de que la reforma es en sí un ejercicio de renovación de la concepción y de las prácticas de gobernanza. Al buscar renovar la gobernanza, los reformadores esperan dotarse de instrumentos suficientes y eficaces para alcanzar los objetivos anunciados en las reformas. Sin pretender un análisis histórico exhaustivo, nuestro trabajo demuestra que las concepciones y las modalidades operativas se transformaron a través del tiempo y que ellas revelan ciertas tensiones inherentes al proceso de transformación y de legitimación de los sistemas públicos de salud. El discurso pragmático que parece estar en el núcleo de 
los proyectos actuales de reforma da cuenta de una situación particularmente delicada. En efecto, pese a los fuertes y recurrentes disentimientos ideológicos sobre la necesidad de mantener o no la integralidad de un sistema público de salud deberán encontrarse algunas vías de mejora del sistema.

Desarrollamos nuestro análisis en cuatro etapas. La primera sección discute las relaciones entre reforma y cambio. La segunda describe la concepción de la gobernanza subyacente a nuestro análisis. La tercera sección traza, mediante un análisis de contenido de diversos informes que proponen reformas, la evolución del concepto de gobernanza en los sistemas de salud en Canadá. Finalmente, discutimos el alcance de la llamada perspectiva "gerencial" de gobernanza, que creemos es la dominante en los proyectos actuales de reforma.

El análisis que desarrollamos considera el sistema de salud como un sistema de acciones complejo poco permeable a intervenciones que apunten a transformarlo en profundidad. El ejercicio de la gobernanza se hace pues esencialmente sobre el modo de la negociación y de la transacción, y debe tomar constantemente en cuenta por una parte, las múltiples interdependencias a la base del funcionamiento del sistema de salud, y por otra, la pluralidad de ideologías que definen los proyectos reformadores y son, a la vez, vehiculados por ellos.

\section{LAS REFORMAS Y EL CAMBIO}

La noción misma de reforma supone que se percibe una necesidad de influir las orientaciones o el funcionamiento de un sistema. Las presiones a favor del cambio provienen de diferentes orígenes. Los cambios demográficos, la evolución de los conocimientos científicos y de las tecnologías, y las transformaciones en el entorno político y económico de los sistemas de salud, provocan una fuerte demanda por el emprendimiento de reformas (3). Más específicamente, esas presiones conducen a focalizar diferentes zonas de mejora del funcionamiento del sistema de atención: la atención primaria o de primer nivel (4), el estatuto y el rol del hospital (5), la atención y los cuidados domiciliarios y los mecanismos de cobertura de los costos y de la gestión del uso de los medicamentos (6-8). La implementación de las transformaciones en diferentes sectores de la oferta de atención reposa sobre varios factores, entre los que se destacan la nivelación de las plataformas técnicas y de inmovilización y la introducción de tecnologías innovadoras, recursos humanos suficientes, con la experiencia y las habilidades requeridas para asumir sus roles diferencialmente, así como inversiones en materia de gestión de las 
informaciones que permitan apoyar los esfuerzos de renovación de la oferta de atención. Las reformas emprendidas en el área de la salud se asemejan, según el caso a proyectos de cambio más o menos ambiciosos y complejos. Las reformas pueden inscribirse en continuidad con los principios fundamentales y organizadores de un sistema, o incluso buscar establecer una ruptura con estos últimos. La implantación en 2002 de grupos de medicina familiar (GMF) en Québec que tuvo como objetivo ofrecer una atención de proximidad más continua y accesible, es una reforma que se inscribe en continuidad con los fundamentos y modos de organización prevalentes en el sistema de salud. La reforma actual del sistema de salud de Québec apunta a operar un cambio más importante, puesto que intenta institucionalizar el principio de responsabilidad poblacional, subordinar ese principio a una gobernanza gerencial y reorganizar la producción de la atención y de los servicios en función de las necesidades de los usuarios más que de los intereses inmediatos de los establecimientos y de los proveedores de servicios. Desde nuestra perspectiva, la presión para renovar la gobernanza del sistema de salud, será tanto mayor cuanto más radicales sean los cambios que busque realizar la reforma emprendida.

El sistema de salud enfrenta así numerosas e importantes contingencias que lo estimulan a transformarse, y la voluntad de aportar cambios al funcionamiento de esos sistemas no puede ser asimilada totalmente a posiciones ideológicas o a la defensa de intereses particulares. Debido a los recursos que moviliza y a la importancia que la población otorga a la atención de la salud, el sector de la salud es una meta priorizada los reformadores, en el conjunto de los países de la OCDE (9). La reforma es, en cierta manera, la expresión de la voluntad del Estado de actuar sobre un sistema del cual es responsable y cuyas deficiencias percibidas plantean problemas (10). Tal constatación sobre la necesidad de las reformas no implica que ellas sean guiadas únicamente por un principio de bien público. Puesto que ellas constituyen un asunto privilegiado de intervención del Estado en la construcción de las instituciones sobre las que éste tiene la responsabilidad, las reformas ofrecen una ocasión privilegiada para diferentes grupos de imponer sus visiones o de defender sus intereses particulares. Los recurrentes debates sobre el rol y el lugar de lo privado en el sistema de salud canadiense son bien reveladores a este respecto, y oponen visiones contrapuestas del rol del Estado y de la legitimidad de la intervención pública $(11,12)$. De hecho, las reformas parecen más necesarias que nunca dando lugar a luchas entre grupos en torno a la materialización de las ideas en la sociedad (13). 
A los fines de este trabajo, consideramos las reformas como un ejercicio de conducción del cambio a gran escala, lo cual que implica la movilización de un conjunto de actores y de organizaciones con valores, objetivos y proyectos más o menos compatibles o convergentes. Este cambio puede ser progresivo o radical. La reforma no es en sí la causa de los cambios. Ella apunta a dominar y obtener el mejor partido de las transformaciones que estructuran la evolución de los sistemas de salud. La reforma busca inducir de una manera deliberada una respuesta a esas transformaciones y a actuar sobre la capacidad del sistema de salud para adaptarse y anticipar las transformaciones que se imponen en su entorno. Aquí la gobernanza remite a las capacidades de conducción presentes en el sistema de salud en relación a la concepción e implementación de las reformas.

\section{LA GOBERNANZA Y LAS REFORMAS}

La gobernanza mantiene una doble relación con las reformas. Consiste, ante todo, en la movilización de un conjunto de herramientas (ideas, normas, reglas, recursos, procesos) necesarias para el desarrollo del proyecto de cambio inherente a toda reforma. Remite también a los diferentes instrumentos que son movilizados para transformar la acción pública de manera de asegurar la capacidad de poner en marcha el proyecto de cambio. Nuestro análisis apunta a decodificar las representaciones de la gobernanza vehiculadas en los proyectos de reforma para favorecer la concepción y la implementación de los cambios.

El tema de la gobernanza ha sido objeto de numerosos trabajos en el terreno de la administración pública, de la sociología y de la organización de los servicios de salud (14-19). Estos trabajos prestan una atención explícita a los mecanismos que permiten producir en la sociedad la convergencia entre actores dispersos y los intereses potencialmente divergentes, con el fin de alcanzar objetivos colectivos $(20,21)$. Clásicamente, en la ciencia de la administración, la gobernanza es definida en función de la relación desarrollada entre una organización y sus propietarios. El análisis de la gobernanza versa así sobre la relación de delegación que se opera entre una estructura de autoridad y una organización, y los incentivos económicos susceptibles de hacer productiva esa relación para organizaciones que operan en situaciones análogas a las que prevalecen en el mercado. Si bien esta perspectiva de análisis parece demasiado restrictiva para dar cuenta de los procesos sociales y políticos constitutivos de las prácticas de gobernanza, ella tiene el mérito de poner el énfasis en la relación de delegación que siempre caracteriza los 
vínculos entre aquellos que hacen la promoción de una reforma y los que están encargados de hacerla aplicar $(10,22)$.

Otros trabajos sobre la gobernanza han buscado comprender los cambios que se producen en los métodos de ejercicio del poder y sobre las condiciones que permiten asentarse a una autoridad que sea legítima $(15,16,20,21,24)$. Al ser las reformas un ejemplo de proyecto colectivo, la producción del colectivo y la capacidad de inventar nuevas técnicas de gobierno operan simultáneamente (15) en un contexto donde las formas tradicionales de autoridad son juzgadas inoperantes $(23,25)$. Asimismo, cuando una reforma busca inducir cambios más radicales, la invención de nuevas técnicas de gobierno se torna crucial. El interés desarrollado actualmente por la implementación de nuevos mecanismos de coordinación y de integración de los servicios de salud (26) y por la emergencia de formas de control más distanciadas, como los sistemas de evaluación de resultados (performances) en el sector público, hacen parte de esta búsqueda de una renovación de las técnicas de gobierno (27).

Inspirándose en trabajos de la sociología y del análisis organizacional, Hatchuel aborda explícitamente esta complejización de la gobernanza. Según dicho autor, la gobernanza consiste en "la concepción y la conducta de una acción colectiva a partir de una posición de autoridad" (17). No obstante, el ejercicio de la gobernanza es indisociable de la noción de prospectiva definida como "un proceso de producción... de conocimientos, que versan sobre el devenir de un colectivo, y cuyos mecanismos de aprendizaje se han hecho posibles por una forma de gobernanza" (17).

Siguiendo a Hatchuel, cuatro cambios de gobernanza pueden ocurrir en las reformas: 1. La emergencia de nuevos objetos de gobierno; 2 . La definición de una utopía hacia la que debería tender un sistema; 3. La designación de nuevas figuras de actores, y; 4. La puesta en marcha de una nueva instrumentación. La concepción de la gobernanza avanzada por Hatchuel (1996) tiene la ventaja de ser sensible al imperativo de actuar en sistemas públicos de servicios que tienen problemas importantes de funcionamiento, y a la necesidad de inventar nuevos mecanismos de ejercicio del poder para hacer posible el cambio. Para conducir el cambio, las reformas van así a provocar o intentar inducir importantes modificaciones en las estrategias o los métodos de ejercicio del poder. A título de ejemplo, las reformas pueden favorecer la emergencia de nuevos objetos de gobierno, o dicho de otra manera, favorecer una nueva teoría del sistema de salud. La emergencia de 
una representación del sistema de salud como instrumento para producir la salud de la población, y no únicamente centrado en la cura de la enfermedad es un ejemplo de cambio a nivel del objeto de gobierno. En el área de la salud, la idea de que esta institución encarna una utopía (ej. los valores democráticos de una sociedad, la identidad de un pueblo...) remite a un ideal que puede animar la orientación de una reforma y las opciones que se tomarán en el curso de su puesta en obra. Los proyectos de reforma vehiculizan de esta forma más o menos explícitamente una redefinición de los objetos de gobierno y de las utopías susceptibles de intervenir en el desarrollo de la gobernanza. Las reformas incitan también a nuevos actores a participar de la gobernanza. Los debates en torno al rol de las organizaciones o de actores identificados con el sector privado, la división de roles entre los diferentes grupos de profesionales o la puesta en aplicación de nuevas estrategias de movilización de actores como la consulta pública, son ejemplos muy claros de un llamado a incrementar la participación o a desarrollar una nueva, por parte de ciertos actores. Finalmente, las inversiones en nuevos instrumentos (sistema de información, sistema de evaluación de resultados...) dan cuenta de las ambiciones de renovación de la gobernanza en el curso de las reformas. Estas cuatro dimensiones que juegan en la renovación de la gobernanza apuntan a incrementar la capacidad de actuar en favor del cambio buscando asegurar la adhesión y la movilización de redes de actores heterogéneos para hacer posible una acción pública que calificamos aquí de reforma. La reforma se asemeja así a un proceso de renovación de la acción pública revelado por las transformaciones que ella busca inducir en el ejercicio de la gobernanza: cuanto más ambiciosos son los objetivos de cambio asociados a toda reforma, más parece que la gobernanza deberá renovarse.

\section{LOS PROYECTOS DE REFORMA DEL SISTEMA DE SALUD}

Nuestro análisis se apoya en el examen de informes preparados en el marco de diferentes comisiones que apuntan a reformar los sistemas de salud en Canadá. Para dar cuenta de las nuevas tendencias en materia de gobernanza reveladas por las reformas, analizamos las ideologías y los principios organizadores que se encuentran en el núcleo de los proyectos reformistas. (28). Por ideología entendemos la visión evocada en los proyectos de reforma para garantizar la perennidad o la renovación del sistema de salud. Esta visión es portadora, más o menos explícitamente, de los valores que deben subyacer al sistema de salud, de su misión primera y del ideal que anima su evolución a largo plazo. Ella remite así a la emergencia de nuevos objetos de gobierno y a la utopía que encarna el sistema de salud. Los principios 
organizadores comprenden a la vez la estructura, los roles atribuídos a los diferentes actores, y los mecanismos de gestión y de evaluación del sistema de salud. Esos principios se relacionan más directamente con dos dimensiones de la gobernanza, sea el ingreso de nuevos actores y la implementación de una nueva instrumentación para apoyar el cambio. Debido a las ideologías y a los principios organizadores que ellas evocan, las reformas valorizan formas de gobernanza específicas.

Las formas de gobernanza vehiculadas por las ideologías reformistas. Cinco ideologías pueden ser identificadas en el análisis de los proyectos de reforma de las diferentes provincias canadienses y a nivel del gobierno federal: la ideología democrática, la ideología de la "salud poblacional", la ideología de negocios, la ideología gerencial, y la ideología de la equidad y del humanismo. A título de ejemplo, y sin pretensiones de exhaustividad o de un análisis histórico del tema en profundidad, presentamos algunas observaciones sobre la organización del discurso reformista en esos proyectos, y en qué medida se evocan diferentes concepciones de la gobernanza. Más precisamente, la ideología reformista implica en sí misma uno o varios objetos de gobierno y un ideal hacia el cual el sistema de salud debería tender.

La ideología democrática se apoya sobre el principio de una descentralización política y fiscal con el fin de redistribuir el poder a las comunidades locales $(29,30)$. La ideología de la salud poblacional (31-33) promueve políticas públicas fuertes atacando los determinantes de la salud constituidos por las condiciones de trabajo y de vivienda y las políticas económicas y de educación. La ideología "de negocios" se inspira en el modelo clásico del mercado en economía favoreciendo la competencia entre organizaciones y proveedores de atención y de servicios y el principio de la soberanía del consumidor que debe tener la capacidad de realizar opciones con recursos que son privados. La ideología gerencial está estrechamente ligada al principio de eficiencia descrito por Ferlie y colegas (27). Según esta ideología, es posible mejorar el funcionamiento del sistema de salud otorgando un rol más importante a la gestión y a las medidas incentivadoras y mecanismos de imputabilidad. El equilibrio entre mecanismos burocráticos, políticos y de mercado está en el centro del pragmatismo vehiculado por la ideología gerencial (34). La ideología de la equidad y del humanismo está basada en la búsqueda de una mayor justicia social. La gestión y el financiamiento público del sistema de salud es aquí garante del principio de justicia social que tiene como consecuencia un acceso equitativo para todos a la atención y a los servicios de salud. 
Observamos de plano que las ideologías se manifiestan con una intensidad variable a través del tiempo en los diferentes proyectos de reforma. Además, los proyectos reformistas preparados por las diferentes comisiones se basan en una mezcla de dosis variables de las diferentes ideologías.

A título de ejemplo, la reforma propuesta por la Comisión CastonguayNepveu (35) que presidió la creación del sistema público de salud en Québec, se apoyaba sobre una combinación de ideologías de equidad y humanismo, gerencial, democrática y de salud poblacional. De hecho, la Comisión proponía integrar a la administración pública la organización y la responsabilidad de producir y de dispensar la atención en salud. El objetivo buscado era el de asegurar una accesibilidad a los servicios a todos los ciudadanos y ciudadanas sin distinción de ingreso y lugar. La Comisión valoraba también una sistematización racional de los roles y de la misión de los diferentes componentes del sistema característico de una ideología gerencial. Ella ponía el acento sobre el principio de una co-gestión de la atención y de los servicios por parte de los usuarios, y una descentralización de la decisión asociada a una ideología democrática. Finalmente, por su voluntad de promover la atención y los servicios al exterior de los hospitales, y de una mayor integración de lo social y lo sanitario, ella también hacía lugar a una perspectiva próxima a la de la salud poblacional ${ }^{2}$.

También el informe de la Comisión Rochon (36) se apoyaba sobre un amplio abanico de ideología reformista (democrática, gerencial y con menor importancia de las de salud poblacional y de equidad y humanismo) para reformar el sistema de salud en Quebec. Ese informe abría a una renovación sustancial de la gobernanza. El informe Clair, catorce años más tarde, otorgaba una importancia primera a la ideología gerencial como palanca para la reforma del sistema de salud en Québec y vehiculaba en forma secundaria un discurso de salud poblacional y de búsqueda de la equidad. La importancia acordada a la ideología gerencial traducía una fuerte determinación para renovar la gobernanza a fin de hacer posible el cambio.

Las profundas tensiones entre los defensores de la privatización (ideología de negocios) y aquellos que privilegian el sistema público, han favorecido

\footnotetext{
${ }^{2}$ Para un análisis más completo de las ideologías implícitas y de los principios organizadores priorizados por la Comisión Castonguay-Nepveu, consultar el texto de Renaud M. Les réformes québécoises de la santé ou les aventures d'un État narcissique. En : Bozzini L, Renaud M, Gaucher D, Llambias-Wolff j (Eds.). Médecine et société-Les années 80. Montréal: Éditions Coopératives Albert Saint-Martin; 1981. p. 513-549.
} 
recientemente el surgimiento de una visión pragmática característica de la ideología gerencial en los discursos de los reformadores. Esta tendencia es análoga "a la nueva gestión pública" muy extendida en la escena europea (27). Los proyectos reformistas, con el tiempo serán menos idealistas, con excepción del informe de la Comisión Romanow (1) que afirma sin ambages la importancia de preservar y de adherir a los valores fundamentales de la ideología de la equidad-humanismo. Lo que importa a partir de entonces es la mejora del funcionamiento del sistema de atención con el fin de responder mejor a las expectativas expresadas por la población. El informe de la Comisión Fyke (37) sobre el sistema de salud en Saskatchewan encarna de forma ejemplar el pragmatismo que marca los discursos reformistas recientes. De hecho, en dicho informe, la ideología gerencial predomina totalmente y está al servicio de la obtención de una mayor calidad de la atención. La importancia otorgada en la actualidad en el área de la salud a las prácticas basadas en evidencias empíricas (38) abre la puerta a una influencia aún mayor y a un predominio aún más importante de la ideología gerencial. La representación del sistema de salud que a partir de allí se vehicula es la de una gran empresa guiada por una información racional con vistas a la obtención de un beneficio óptimo de la utilización de los recursos. Por otro lado, la ideología del negocio es más visible que antes en ciertos proyectos de reforma, entre ellos el informe Mazankowski (39) para Alberta, y Kirby (40) a nivel del gobierno federal. La ideología del negocio se apoya sobre la hipótesis de que una verdadera dinamización de la gestión no puede operarse fácilmente en el marco de un sistema público, y que los gobiernos no podrán proveer a largo plazo los recursos necesarios para responder a las necesidades de la población. También el informe de la Comisión Clair (2) prestaba una atención particular a la cuestión de la viabilidad del sistema público de salud, puesta en peligro por un aumento de los gastos más allá de la disponibilidad de los recursos colectivos.

En su conjunto, el análisis de las ideologías que nutrieron el trabajo de las diferentes comisiones sobre el futuro de los sistemas de salud en Canadá revela la emergencia de nuevos objetos de gobierno. Por ejemplo, la ideología de la salud poblacional y la ideología democrática ocuparon un lugar significativo en varios proyectos reformistas. Esas dos ideologías convocan a una transformación radical del sistema de salud, y por consiguiente, de su gobernanza para asegurar y regular la participación pública y producir la salud a escala de las colectividades. Por otra parte, las ideologías que tienen a primera vista un potencial más elevado de conmoción de los modos de funcionamiento del sistema de salud ("salud poblacional", "democrática") 
siempre han coexistido con una ideología gerencial que refleja la importancia acordada a una mejora del funcionamiento del sistema de salud. Recientemente, incluso, han cedido terreno a la ideología gerencial. Si las ideologías más "innovadoras" o audaces empujan las reformas al exterior de las fronteras del sistema de salud (políticas públicas en otros sectores, democratización política...), la ideología gerencial invita a una renovación de los objetos y métodos de gobierno al interior mismo del sistema de salud al favorecer, como veremos, el desarrollo de una instrumentación de la gestión, la formación de gestores y la utilización de evidencias científicas al servicio de una mayor eficiencia y eficacia en la utilización de los recursos. En los últimos tiempos, las utopías han ocupado aparentemente un lugar menos importante en los discursos reformistas. La instauración de los sistemas públicos de salud respondía en sus inicios a un ideal de equidad de acceso a la atención y los servicios, y en ciertos casos (Québec) de apertura a una menor medicalización de las intervenciones. La legitimidad del sistema público de salud parece depender cada vez más de su capacidad para proveer la atención y los servicios en función de las necesidades percibidas por los profesionales y los usuarios que de su encarnación en una utopía portadora de valores fundamentales. Las iniciativas tomadas luego del informe Romanow (1) sobre el futuro del sistema de salud en Canadá muestran además una preocupación creciente por una gestión racional del sistema de salud y una apelación menos visible a compartir valores fundamentales.

Formas de gobernanza vehiculadas por los principios organizadores asociados a los proyectos de reformas. La promoción de diferentes ideologías reformistas implica la valorización de una diversidad de principios para reorganizar el sistema de salud. Los proyectos de reforma no pueden contentarse únicamente con proponer una visión de lo que debería ser el sistema de salud. También tienen que enunciar pistas para reorganizar el sistema. Los principios organizadores remiten a elementos importantes de la gobernanza como el rol otorgado a distintos actores en la conducción de la reforma y los métodos retenidos o privilegiados para el ejercicio del poder.

A título ilustrativo, la ideología democrática ha estado asociada a la promoción de políticas de descentralización y más concretamente a la creación de entidades regionales fuertes (Comisión Castonguay-Nepveu y Comisión Rochon en el caso del sistema de salud quebequense). En Québec, la regionalización era percibida en su origen como un poderoso instrumento para incrementar la participación de la población en la gobernanza del sistema de salud. Las reformas actuales muestran que es difícil consolidar 
innovaciones sobre el plano de la gobernanza, particularmente cuando exigen de parte del gobierno central que permita la emergencia de políticas locales o regionales autónomas en materia de organización de la atención y los servicios (41). La ideología de la " salud poblacional " por su lado dio lugar a la valorización de principios organizadores que a primera vista son contradictorios. Ella favorece el desarrollo autónomo de programas y de intervención en las comunidades. El financiamiento de iniciativas de promoción de la salud en $\mathrm{CLSC}^{3}$ y en las direcciones regionales de salud pública en Québec opera en el sentido de un desarrollo de programas de intervención arraigados en comunidades locales. La ideología de la salud de las poblaciones también está asociada a la promoción de políticas públicas dirigidas por un gobierno central fuerte, que busca actuar sobre indicadores de salud considerados desfavorables.

Los informes recientes de diferentes comisiones muestran que los principios organizadores del sistema de salud se articulan cada vez más entorno a estrategias que apuntan a dinamizar la gestión (gestión descentralizada, contractualización, medida de los resultados asociaciones público-privadas, definición de sectores prioritarios, promoción de la utilización de una información racional o científica-ver entre otros, los informes Clair y Arpin y los actuales discursos reformistas sobre la salud en Québec). Empero, esos principios organizadores no son totalmente nuevos. La voluntad de establecer una gestión centrada en los resultados y de desarrollar una instrumentación apropiada para regular el sistema de salud está presente desde hace más de quince años en Québec y ha preocupado varios reformadores por alinear la producción de atención y de servicios con las necesidades de una población más que con los intereses de los proveedores (Ver entre otros el informe de la Comisión Rochon, Gobierno de Québec, 1988).

A nivel federal dos grandes principios organizadores del sistema de salud se enfrentan: un sistema integrado sobre el plan de los valores y con una conducción rigurosa de las operaciones en función de la obtención de ciertos objetivos (informe de la Comisión Romanow), y un sistema cuyo funcionamiento importa mejorar a cualquier precio sin voluntad de afirmar su singularidad o su especificidad (informe Kirby). El informe Romanow

\footnotetext{
${ }^{3}$ Nota de la Traductora: Los CLSC, organismos de servicios de proximidad, son los Centros Locales de Servicios Comunitarios que han sido reemplazados actualmente por los llamados CSSS, Centros de Salud y Servicios Comunitarios.
} 
promueve una visión más centralizada de la gobernanza del sistema de salud. Las recomendaciones contenidas en ese informe dieron lugar a la creación de instituciones, tales como el Consejo Nacional de la Salud, que apuntan a incrementar la utilización de una información racional y a valorizar normas en materia de resultados (performances) para el conjunto de los sistemas de salud de las distintas provincias canadienses.

De esta manera, en el plano de los principios organizadores, la evocación de estrategias y de medios vinculados a la ideología gerencial se ha reforzado a través del tiempo. Esta tendencia puede acompañarse o no de la promoción de principios organizadores vinculados al mundo del sector privado. (ej. : el recurso al mercado, a la competencia, una reivindicación para autorizar el ingreso de nuevos actores del mundo de la economía privada). La promoción de una ideología de negocios favorece la importancia del rol de los gestores) como artífices de las reformas y de la conducción del cambio. Ello estimula también una cierta personificación de la acción al valorizar la figura del empresario en el sector público. Finalmente, la valorización de la ideología gerencial no implica, en el plano organizacional, el rechazo total de las otras ideologías reformistas sino su integración en un marco más restrictivo. La ideología de la salud poblacional, en el plano organizacional va a asumir la forma de una atención particular a ciertos programas preventivos en nombre de la búsqueda de una mayor eficiencia y del logro de ciertos resultados. La ideología democrática va a reflejarse en la promoción de mecanismos que permitan a los ciudadanos actuar como consumidores informados en el mercado de la atención en salud, tal como la promoción y difusión de información sobre los resultados alcanzados por los organismos de salud. De esta forma se otorga una atención particular a la reactividad del sistema.

\section{DISCUSIÓN Y CONCLUSIÓN}

Inicialmente propusimos que la renovación de la gobernanza podía manifestarse por la evocación de nuevos objetos de gobierno, por el recurso a una utopía, por la aparición de nuevas figuras de actores y la puesta en marcha de nuevos instrumentos. Es a través de esas diferentes dimensiones de la gobernanza que se transforman los métodos de ejercicio del poder. Para decodificar las transformaciones de la gobernanza, analizamos las ideologías y los principios organizadores que son vehiculados en los diferentes proyectos de reforma. Éstos se han apoyado fuertemente sobre la evocación de nuevos objetos de gobierno (la calidad de la atención, la salud poblacional, el gerenciamiento de las organizaciones, la democracia...). No obstante, 
resulta difícil asociar a estos objetos de gobierno los medios y los métodos de ejercicio del poder que permiten materializarlos. Aún si la ideología gerencial parece ejercer cada vez más influencia, actualmente es difícil reunir las condiciones que permitan un despliegue acentuado de esta lógica en el sistema de salud, y por consiguiente la puesta en marcha de transformaciones importantes. Basta con pensar en la propensión de los decisores políticos a intervenir directamente en el funcionamiento del sistema de salud, o en la fragilidad de la autoridad gerencial en ese tipo de organización para comprender los desafíos de su aplicación. Además, los desafíos que plantea el desarrollo de una instrumentación apropiada y reconocida como legítima en varias áreas de la acción pública permanecen siendo importantes (42).

Este análisis de nuevas tendencias de la gobernanza reveladas por las reformas nos lleva de hecho a las condiciones para el ejercicio de un poder legítimo en áreas caracterizadas por un grado de pluralismo elevado como el de la salud (43). Varias de las ideologías reformistas propuestas (salud poblacional, democratización) no han tenido un anclaje sólido en las lógicas que estructuran el sistema de salud. Esas ideologías son difíciles de implementar, más aún porque las presiones actuales militan en favor de una mejora de la dispensa de atención. Por otra parte, y a pesar de los desafíos que ella implica, la ideología gerencial ha tomado últimamente un lugar más relevante, y ella puede favorecer la implantación de pequeños cambios progresivos en los organismos de salud. Nuestros trabajos sobre las reformas han mostrado que para que las transformaciones se produzcan, es necesaria una conjunción entre los efectos que vienen de lo alto, y las iniciativas que emergen de la base de las organizaciones $(16,44)$. La gobernanza gerencial ofrece pistas para articular mejor las dinámicas en los distintos niveles del sistema de salud. Puede conducir a negociar los proyectos de cambios y también a estimular la adhesión de los profesionales haciendo un uso crítico de las distintas herramientas susceptibles de producir el cambio. En su análisis de las reformas de la administración pública, Brunsson y Sahlins-Andresson (45) observan que varias de esas iniciativas tienen por objeto crear organizaciones completas, es decir, organizaciones cuyo funcionamiento no estaría dominado principalmente por lógicas jerárquicas ni políticas. Las organizaciones públicas así reformadas se asemejarían a empresas donde el gerenciamiento juega un rol de primer plano. Las reformas tendrían por ambición instaurar dicha gobernanza gerencial. La promoción que se hace actualmente de la ideología gerencial puede constituir también una fuerza de representación para estimular a las autoridades públicas y burocráticas a intervenir en forma diferente en la gobernanza del sistema de salud. Sería 
necesario analizar más en profundidad el rol que pueden jugar los métodos propios del gerencialismo para contribuir a la actualización de ideologías reformistas que pertenecen a otros universos. A este respecto, la ideología de la salud poblacional necesita también una instrumentación que le permita conducir eficazmente los programas de intervención o de políticas públicas favorables a la salud. La ideología democrática también tiene necesidad de un aparataje organizacional que permita la puesta en marcha de procesos eficaces de participación y de movilización del público. En los dos casos, los decisores y los profesionales deben poder ser imputables de objetivos que están lógicamente asociados a la promoción de una ideología reformista particular. La gobernanza gerencial puede contribuir eventualmente a repensar las dinámicas de imputabilidad a favor de una ideología más audaz o innovadora.

Nuestro análisis sugiere que es importante comprender mejor los procesos de renovación de la gobernanza para poder evaluar el potencial de las reformas que se emprenden o emprenderán en el campo de la salud

\section{REFERENCIAS}

1. Gouvernement du Canada. "Guidé par nos valeurs: l'avenir des soins de santé au Canada". Rapport final, Ottawa (Rapport Romanow); 2002.

2. Gouvernement du Québec. "Les solutions émergentes". Rapport de la Commission d'étude sur la santé et les services sociaux ,Québec, Éditeur officiel (Rapport Clair); 2001.

3. Contandriopoulos AP. Pourquoi est-il si difficile de faire ce qui est souhaitable? Gestion. 2002; 27(3):142-150.

4. Wilson R, Shortt, SED, Dorland J. Implementing Primary Care Reform. Montreal \& Kingston: McGill-Queen's University Press; 2004.

5. Denis JL. Governance and Management of Change in Canada's Health System, Changing Health Care in Canada. Forest PG, Marchildon GP, Mclntosh T (eds.). Toronto: University of Toronto Press; 2004. p. 82-114.

6. Sinclair D. After Kirby and Romanow: Where to From Here. Hospital Quarterly 2002; 6(2): 42-43.

7. Morgan SG, Willison DJ. Post-Romanow Pharmacare: Last-Dollar First. First-Dollar Lost? Healthcare Papers 2004; 4(3): 10-20.

8. Sketris IS, Brown MG, Murphy AL. Policy Choices for Pharmacare: The Need to Examine Benefit Design, Medication Management Strategies and Evaluation Approaches. Healthpapers. 2002; 4 (3):36-45.

9. Saltman R, Busse R, Mossialis E (eds.), Regulating Entrepreneurial Behaviour in European Health Care Systems. European Observatory on Health Care System Series. Buckhingham: Open University Press; 2002.

10. Rocher G. Le "laboratoire" des réformes dans la Révolution Tranquille. Conférence Desjardins McGill University's Quebec Studies Program. November 6. Montreal: McGill University; 2001.

11. Chodos H, MacLeod JJ. Romanow and Kirby on the Public/Private Devide in Healthcare: Demystifying the Debate. Healthcare Papers. 2004; 4 (4) 2004: 10-25. 
12. Marchildon GP. The Public/Private Debate in the Funding, Administration and Delivery of Healthcare in Canada. Healthcare Papers. 2004; 4 (4) 2004: 61-68.

13. Maioni A. From Cinderella to Bell of the Ball: The Politics of Primary Care Reform in Canada. In: Wilson R, Shortt SED, Dorland J (eds.). Implementing Primary Care Reform. Montreal \& Kingston, McGill-Queen's University Press. 2004:97-110.

14. Kooiman J (Ed.). Modern Governance, Newbury Park. Sage; 1993.

15. Chevalier J. Présentation La gouvernabilité, Paris, PUF; 1996: 5-16.

16. Simon H. Public administration in today's world of organizations and markets. Political Science and Politics. 2000; 33(4):749-56.

17. Hatchuel A. Prospective et gouvernance: quelle théorie de l'action collective? Prospective pour une gouvernance démocratique (coord.: Édith Heurgon et Josée Landrieu), Centre culturel international de Cerisy-La-Salle; 2000. p. 29-42.

18. Harding A, Preker AS. A conceptual frameworkd for the organizational reforms of hospitals. In: Preker AS, Harding A (Eds.). Innovations in Health Service Delivery Washington, D.C.: World Bank; 2003. p. 23-78.

19. Saltman RB, Ferroussier DO. The concept of stewardship in health policy. Bulletin of the Wolrd Health Organizations. 2000; 78(6), 732-39.

20. March JG. The War is Over and the Victors have Lost. Journal of Socio-Economics. 1992; 21: 362-367.

21. Marsh D, Rhodes RAW. Policy Networks in British Politics: A Critique of Existing Approches. In: Marsh D, Rhodes RAW (eds). Policy Networks in British Government. Oxford: Clarendon Press ; 1992. p. 1-26.

22. Denis JL, Valette A. Changement de structure de régulation et performance du mandat. Finance, contrôle et stratégie. 2000; 3(2): 57-79.

23. Lascoumes P. Rendre gouvernable: de la "traduction" au "transcodage ". L'analyse des processus de changement dans les réseaux d'action publique. La gouvernabilité, Paris, PUF. 1996; 325-338.

24. Klijn EH. Analysing and Managing Policy Processes in Complex Networks: A Theoretical Examination of the Concept Policy Network and its Problems. Administration and Society. 1996; 28(1): 90-119.

25. Callon $\mathrm{M}$, Lascoumes $\mathrm{P}$, Barthe $\mathrm{Y}$. L'action mesurée ou comment décider sans trancher (Chap. 6), Agir dans un monde incertain, essai sur la démocratie technique. Paris: Seuil. 2001; 263-308.

26. Shortell SM, Gillies RR, Anderson DA, Erickson EM, Mitchell JB. Remaking health care in America: Building organized delivery systems. San Francisco: Jossey-Bass; 1996.

27. Ferlie L, Lynn CP (ed). Handbook of Public Administration: London, UK: Oxford University Press; 1996. p. 446-467.

28. Hinings CR, Greenwood R. The Dynamics of Strategic Change. Oxford: Blackwell; 1988.

29. Dickinson HD. How Can the Public Be Meaningfully Involved in Developing and Maintaining an Overall Vision for the Health System Consistent with Its Values and Principles? Discussion paper \#33. Commission on the Future of Health Care in Canada; 2002.

30. Hirschman AO. Exit, Voice and Loyalty. Cambridge: Harvard University Press; 1970.

31. Hayes MV, Dunn JR. Population Health in Canada: A Systematic Review. CPRN Study No. H01. Canadian Policy Research Networks Inc.; 1998.

32. Evans RG, Barer ML, Marmor TR. (eds.). Why are Some People Healthy and Others Not? The Determinants of Health of Populations, Hawthorne, NY: Aldine de Gruyter; 1994.

33. Evans R. Interpreting and addressing inequalities in health: from Black to Acheson to Blair to? London: Office of Health Economics; 2002.

34. Heath J. The Efficient Society, New-York: Penguin Books; 2001.

35. Gouvernement du Québec. Rapport de la Commission d'enquête sur la Santé et le Bienêtre social, Québec, Éditeur officiel (Rapport Castonguay-Nepveu); 1973. 
36. Gouvernement du Québec. Rapport de la Commission d'enquête sur les services de santé et les services Sociaux. Québec, Éditeur officiel (Rapport Rochon); 1988.

37. Government of Saskatchewan. Caring for Medicare : Sustaining a Quality System. Commission on Medicare report (rapport Fyke); 2001.

38. Walshe K, Rundall TG. Evidence-based management: From theory to practice in health care. The Milbank Quarterly. 2001; 79(3):429-457.

39. Government of Alberta. A Framework for Reform. Report of the Premier's Advisory Council on Health (rapport Mazankowski); 2001.

40. Kirby MJL. La santé des Canadiens -"Le rôle du gouvernement fédéral" - Volume six: Recommandations en vue d'une réforme, Comité sénatorial permanent des Affaires sociales, des sciences et de la technologie; octobre 2002.

41. Contandriopoulos D, Denis J.L. Langley A, Valette A. "Governance Structures and Political Processes in a Public System". Public Administration. 2004; 82 (2):627-655.

42. Townley B, Cooper DJ, Oakes L. Performance Measures and the Rationalization of Organizations. Organization Studies. 2003; 24 (7): 1045-1072.

43. Denis JL, Langley A, Rouleau L. Rethinking Leadership in Public Organizations. Oxford Handbook of Public Administration; 2005.

44. Denis JL, Lamothe L, Langley A. Government-funded experiments as resources for renewal in health care? Paper presented Academy of Management Conference. Washington; August 2001.

45. Brunsson N, Sahlin AK. Constructing organizations: The example of public sector reforms. Organization Studies. 2000; 21(4): 721-46. 